\title{
Influenza infection and Kawasaki disease
}

\author{
Xijing Huang ${ }^{[1]}$, Ping Huang $\left[{ }^{[1]}\right.$, Li Zhang ${ }^{[1]}, X_{i a o f e i} X e^{[1]}$, Shuliang Xia ${ }^{[1]}$, \\ Fang Gong ${ }^{[1]}$, Jia Yuan ${ }^{[1]}$ and Liling Jin ${ }^{[1]}$
}

[1]. Heart Center, Guangzhou Women and Children's Medical Center, Guangzhou Medical University, Guangzhou, China.

\begin{abstract}
Introduction: The objective of this study was to investigate the possible link between influenza (Flu) infection and Kawasaki disease (KD). Methods: We examined the medical records of 1,053 KD cases and 4,669 influenza infection cases hospitalized at our institute from January 1, 2011 to December 31, 2013. Cases of KD with concomitant influenza infection formed the KD + Flu group. Each $\mathrm{KD}+$ Flu case was matched with $2 \mathrm{KD}$ cases and 2 influenza infection cases, and these cases were assigned to the $\mathrm{KD}$ group and Flu group, respectively. The differences in the principal clinical manifestations, course of disease, incomplete KD rate, intravenous immunoglobulin (IVIG) resistance rate, and echocardiographic detection results between the KD + Flu group and KD group were compared. The fever durations and laboratory test results of these three groups were compared. Results: 1) The seasonal variations of the KD + Flu group, KD group and Flu group were similar. 2) The morbidity rate of incomplete $\mathrm{KD}$ was higher in the KD + Flu group compared with the KD group. 3) Patients in the KD + Flu group exhibited a longer time to KD diagnosis compared with patients in the KD group. 4) The KD + Flu group exhibited the longest fever duration among the three groups. 5) The CRP and ESR values in the KD + Flu group were higher those in the Flu or KD groups. Conclusions: Concomitant influenza infection affects the clinical manifestations of KD and can impact the laboratory test results and the diagnosis and treatment of the disease. However, it remains unclear whether influenza contributes to KD etiology.
\end{abstract}

Keywords: Kawasaki disease. Influenza. Influenza virus.

\section{INTRODUCTION}

Kawasaki disease (KD) is a systemic vasculitis that predominantly affects infants and young children that are 6 months to 5 years old and has become the leading cause of acquired heart disease among children ${ }^{(1)}$. Although many studies have been performed since the first description of KD in 1967, the etiology of the disease remains unclear, and there is currently no specific laboratory diagnostic test for $\mathrm{KD}^{(2)}$. Some researchers have reported that the incidence of $\mathrm{KD}$ has increased in recent years ${ }^{(3)}{ }^{(4)}$. A large number of epidemiological and clinical observations have indicated that infectious microorganisms might contribute to KD. Human adenovirus, mycoplasma, streptococci, retroviruses, Epstein-Barr virus, and parainfluenza type 3 have been implicated in the etiology of $\mathrm{KD}^{(5)(6)(7)(8)(9)(10)}$. In this present study, we explored whether the influenza virus represents a candidate KD pathogen through a retrospective study.

Corresponding author: Ping Huang. Heart Center/Guangzhou Women and Children's Medical Center, Guangzhou Medical University. Middle Ren Min Road 318, Yuexiu District, 510000 Guangzhou, China.

Phone: 8620 8133-0675

e-mail: huangp9999@126.com

Received 18 March 2015

Accepted 18 May 2015

\section{METHODS}

The medical records of $1,053 \mathrm{KD}$ patients and 4,669 influenza infection patients that were hospitalized from January 1, 2011 to December 31, 2013 at our institute (Guangzhou Women and Children's Medical Center, Guangzhou, China) were collected. The diagnosis of $\mathrm{KD}$ was made according to the 2004 American Heart Association (AHA) guidelines. Patients with 2 to 3 principal clinical features of KD and unexplained fever for 5 days as well as abnormal laboratory values were classified as exhibiting incomplete $\mathrm{KD}^{(11)}$. Patients with persistent or recrudescent fever in the 48 hours following intravenous immunoglobulin (IVIG) treatments were defined as IVIG-resistant cases ${ }^{(12)}$. It should be noted that patients with influenza only were not treated with IVIG.

\section{Routine and biochemical detection}

Kawasaki disease patients underwent blood tests for the following parameters on the first day of hospitalization before receiving treatment: white blood cell (WBC), polymorphonuclear leukocytes (PMN), C-reactive protein (CRP), erythrocyte sedimentation rate (ESR), albumin (ALB), alanine aminotransferase (ALT), aspartate aminotransferase (AST), and creatine kinase isoenzyme (CK-MB).

\section{Pathogen detection}

Hospitalized patients underwent serologic IgM tests for the detection of respiratory pathogens (mycoplasma, chlamydia, 
influenza A and B, adenovirus, respiratory syncytial virus, and Epstein Barr virus) by immunofluorescence assay (IFA) and pharyngeal swab tests for the detection of influenza virus, adenovirus, respiratory syncytial virus and enteric viruses by fluorescent polymerase chain reaction technology. Patients with diarrhea were tested for rotavirus by reverse transcription polymerase chain reaction (RT-PCR) and for Shigella using a coagglutination assay.

\section{Grouping and observation index}

$K D+$ Flu group: KD patients positive for influenza A or/ and $\mathrm{B}$ virus but no other detectable pathogens comprised the $\mathrm{KD}+$ Flu group. Fifteen patients were classified as KD + Flu cases, including 9 boys and 6 girls aged 2 months to 4 years with a mean age of 2 years.

KD group: each KD + Flu case was matched with 2 pathogen-negative KD patients with respect to age, gender, and date of hospitalization ( \pm 3 weeks). The match cases included 18 boys and 12 girls aged 4 months to 4 years with a mean age of 1.9 years. These patients comprised the KD group.

Flu group: each $\mathrm{KD}+$ Flu case was matched with 2 patients positive for influenza A or/and B virus but no other detectable pathogens with respect to age, gender, and date of hospitalization ( \pm 3 weeks). The matched cases included 18 boys and 12 girls aged 5 months to 4 years with a mean age of 2 years. These patients comprised the Flu group.

The above three groups were included in our independent sample design. The age, sex and hospitalization time of two latter groups were matched with the $\mathrm{KD}+$ Flu group for comparability. Differences in the clinical manifestations, course of disease, incomplete KD rate, IVIG resistance rate, and coronary artery complications rate between the $\mathrm{KD}+$ Flu group and $\mathrm{KD}$ group as well as the fever duration and laboratory test results of these three groups were compared using statistical methods.

Descriptive analysis was performed using frequency distributions. Means, standard deviations, medians and interquartile ranges (IQ range) were used to summarize the patients' demographic and baseline characteristics. Fisher's exact test was used for qualitative variables as appropriate. The KruskalWallis analysis was used for continuous variables; when the null hypothesis was rejected, we use Bonferroni for multiple comparisons.

A 2-tailed $p$ value $\leq 0.05$ was considered significant for comparisons among the three groups. A 2-tailed $\mathrm{p}$ value $\leq 0.0167 \%(Z>2.93)$ was considered significant for comparisons between any two of the three groups. The Statistical Package for the Social Sciences 19.0 (IBM SPSS 19.0) was used for data analysis.

\section{RESULTS}

Of the 1,053 total KD cases, 705 patients underwent tests for common respiratory pathogens. Of these, 34 cases were positive for influenza virus, including 23 influenza $A$ infections and 11 influenza $\mathrm{B}$ infections. The rate of $\mathrm{KD}$ with concomitant influenza was $4.8 \%(34 / 705)$. Among these 34 patients, 11 patients were only infected with influenza $A$ virus, 4 patients were only infected with influenza B virus, and 19 patients were also infected other pathogens, including mycoplasma, chlamydia, adenovirus, respiratory syncytial virus and Epstein Barr virus. The seasonal variations of the three groups are presented in Figure 1, Figure 2 and Figure 3. As the figures demonstrate, the following common trends were observed: 1) The number of patients steadily increased from January to April and then declined. 2) The number of patients observed in the spring and summer accounted for a larger proportion of the total cases. Moreover, when analyzing the number of patients each month using Pearson's correlation analysis, we observed that the correlation between the KD group and the KD + Flu group was significant $(r=0.92$, $\mathrm{p}$ value $<0.01)$, which partly supports their relationships with respect to seasonal variation.

The clinical manifestations of influenza patients in the Flu group included fever, runny nose and stuffy nose. The principal clinical manifestations of the KD patients included fever, bilateral conjunctival injection, mucosal changes, polymorphous rash, changes in the extremities and cervical lymphadenopathy. Therefore, we were only able to compare the duration of fever between the three groups. The mean fever durations (SD) of the $\mathrm{KD}+$ Flu group, KD group, and Flu group were 9.13h (4.98), 6.4h (1.76) and 3.2h (1.21), respectively. The fever durations of the KD + Flu group and KD group were longer than that of the Flu group ( $\mathrm{p}$ value $<0.01$, $\mathrm{p}$ value $<0.01$ ).

In addition to the influenza symptoms, patients in the $\mathrm{KD}$ + Flu group exhibited other changes in KD symptoms. We summarized and compared the clinical presentation, clinical course and outcomes of the KD + Flu group and $\mathrm{KD}$ group in Table 1. The morbidity rate of incomplete $\mathrm{KD}$ was higher in the $\mathrm{KD}+$ Flu group compared with the KD group ( $40 \%$ vs. $10 \%$, $\mathrm{p}$ value $=0.04$ ). Bilateral conjunctival injection was documented more frequently in the $\mathrm{KD}$ group than in the $\mathrm{KD}+$ Flu group $(100 \%$ vs. $80 \%$, p value $=0.03)$. Furthermore, the time required to diagnose patients in the $\mathrm{KD}+$ Flu group was longer than the time required to diagnose patients with $\mathrm{KD}$ alone $(6 \mathrm{vs} .5 \mathrm{~d}$, p value $=0.03$ ). In summary, atypical symptoms led to an increased rate of incomplete $\mathrm{KD}$, which resulted in a greater time to diagnosis. No statistically difference was observed regarding the occurrence of cardiovascular complications between these two groups.

In addition to CK-MB, the majority of the laboratory values in the three groups summarized in Table 2 were abnormal. The numbers of cases with abnormal CK-MB values were 3,0 and 12 in the $\mathrm{KD}+\mathrm{Flu}, \mathrm{KD}$ and Flu groups, respectively, indicating that CK-MB abnormalities were more common in the KD + Flu group and Flu group than in the KD group ( $\mathrm{p}$ value $<0.05$, $\mathrm{p}$ value $<0.05$, respectively).

\section{DISCUSSION}

The etiology of KD remains unclear, and potential contribution of infections to the etiology of KD is controversial. No consistent association between any infectious agent and KD has been demonstrated. Several scholars have made case reports 


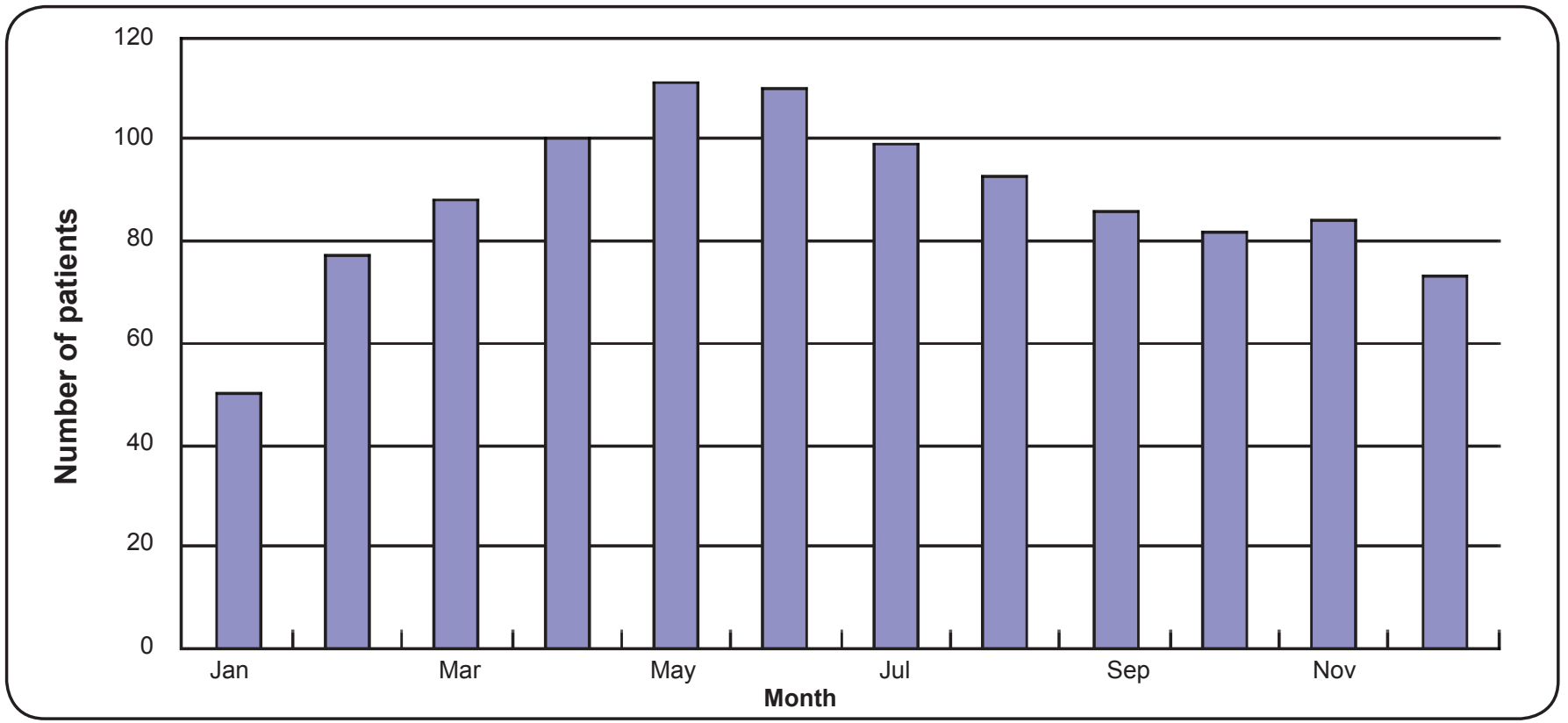

FIGURE 1 - Monthly variation in KD incidence. From January to May, the number of patients with Kawasaki disease gradually increased; the incidence of Kawasaki disease declined slowly from June to December. The number of KD patients presenting in the spring and summer accounted more than $58 \%(610 / 1,053)$ of the patients seen year-long. KD: Kawasaki disease.

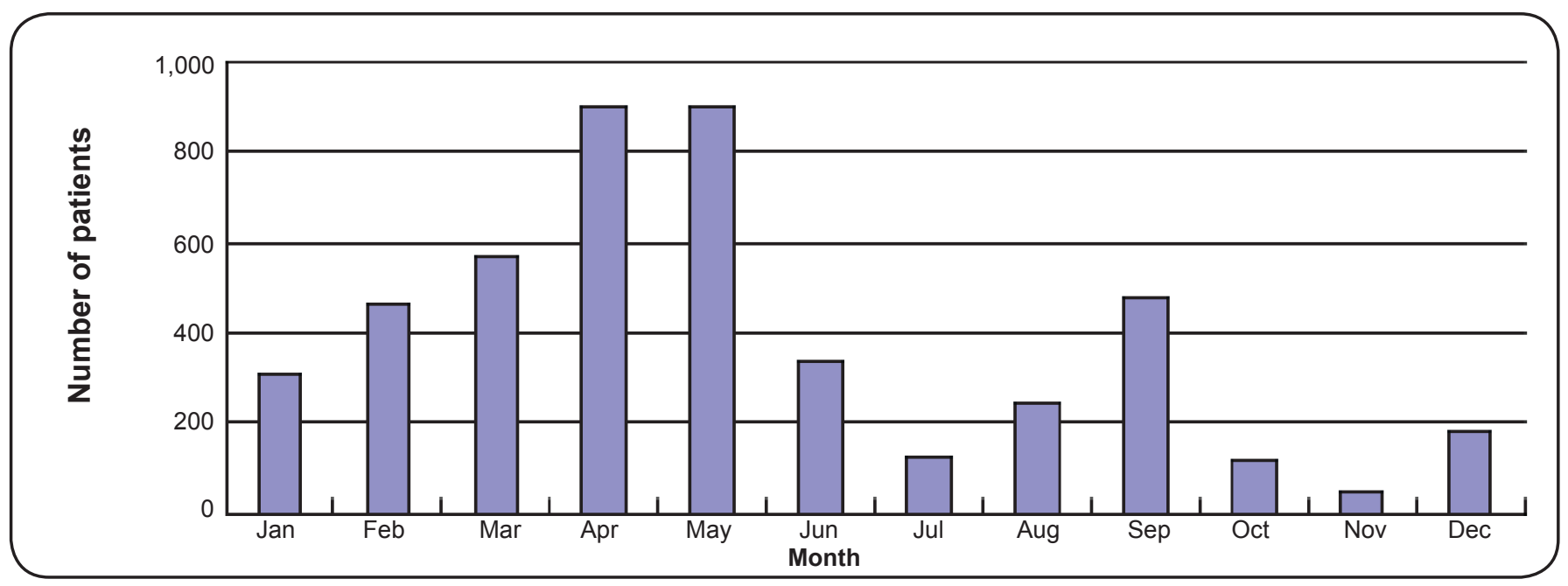

FIGURE 2 - Monthly variation in influenza incidence. From January to May, the number of influenza cases gradually increased. The incidence of influenza declined significantly from June to December and exhibited a small bump in September; the number of influenza patients presenting in spring and summer accounted for more than $65 \%(3,074 / 4,667)$ of the total cases throughout the year.

concerning influenza with concomitant $\mathrm{KD}$, but the details about the impact of influenza on KD are limited ${ }^{(13)}$. In the present study, we used a case-controlled study design with the goal of providing more details regarding the role of influenza in $\mathrm{KD}$.

The rationale for an infectious etiology is largely based on the epidemiologic features of $\mathrm{KD}^{(14)}$. The seasonal variation of KD patients indicates that an infectious pathogen might be relevant to the etiology of $\mathrm{KD}$. In this study, we observed that the epidemiological features of KD and influenza were similar.
First, both KD and influenza affected children aged 6 months to 5 years. Second, like most infectious diseases, these diseases are self-limiting and exhibit similar clinical courses. Third, cases of KD with concomitant influenza tend to cluster temporally and have a predilection for spring and summer. As Figure 1, Figure 2 and Figure 3 demonstrate, the seasonal variation of the KD + Flu, KD and Flu groups are similar, suggesting that there might be a close relationship between KD and influenza. 


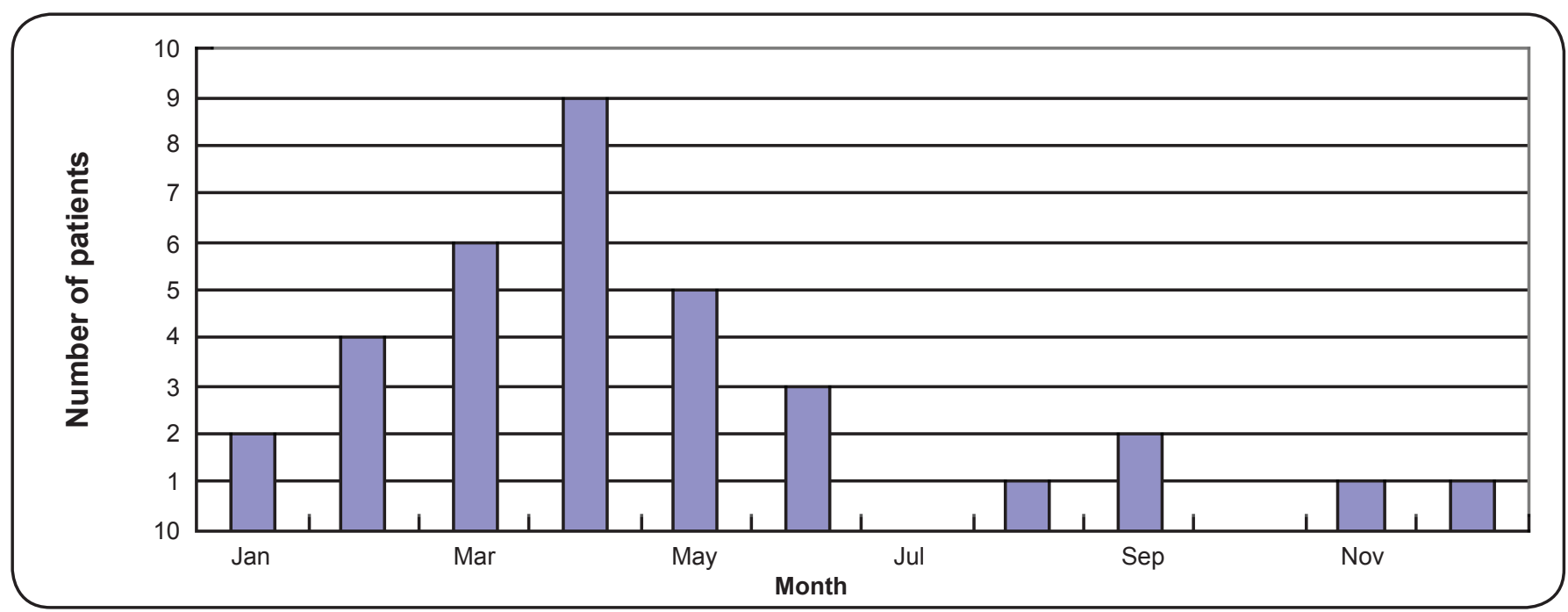

FIGURE 3 - Monthly variations in the incidence of concomitant KD and influenza. From January to April, the number of KD cases with concomitant influenza gradually increased, and the incidence declined significantly from May to December. The number of KD cases with concurrent influenza that presented in the spring and summer accounted for more than $70.6 \%(24 / 34)$ of the cases throughout the year. KD: Kawasaki disease.

TABLE 1 - Demographics, clinical characteristics, and outcomes of the KD + Flu group and KD group.

\begin{tabular}{|c|c|c|c|c|c|}
\hline \multirow[b]{2}{*}{ Variable } & \multicolumn{2}{|c|}{ KD + Flu group $(n=15)$} & \multicolumn{2}{|c|}{ KD group $(n=30)$} & \multirow[b]{2}{*}{$\mathrm{P}$ value } \\
\hline & $\mathrm{n}$ & $\%$ & $\mathrm{n}$ & $\%$ & \\
\hline \multicolumn{6}{|l|}{ Clinical symptoms } \\
\hline mucosal changes & 13 & 86.7 & 29 & 96.7 & $0.25 \#$ \\
\hline polymorphous rash & 11 & 73.3 & 28 & 93.3 & $0.16 \#$ \\
\hline cervical lymphadenopathy & 5 & 33.3 & 17 & 56.7 & $0.21 \#$ \\
\hline \multicolumn{6}{|l|}{ Nonspecific symptoms } \\
\hline flush or desquamation around the anal skin & 7 & 46.7 & 10 & 33.3 & $0.53 \#$ \\
\hline erythema or induration in the bcg vaccination site & 2 & 13.3 & 2 & 6.7 & $0.60 \#$ \\
\hline valvular regurgitation & 0 & 0.0 & 2 & 6.7 & $0.55 \#$ \\
\hline other non-cardiovascular complications & 3 & 20.0 & 4 & 13.3 & $0.67 \#$ \\
\hline \multicolumn{6}{|l|}{ Days required for diagnosis } \\
\hline median (IQ range) & \multicolumn{2}{|c|}{$6(4.5-8)$} & \multicolumn{2}{|c|}{$5(4-6)$} & $0.03 *$ \\
\hline incomplete KD (n, \%) & 6 & 40.0 & 3 & 10.0 & $0.04 \#$ \\
\hline IVIG resistance $(\mathrm{n}, \%)$ & 2 & 13.3 & 5 & 16.6 & $1.00 \#$ \\
\hline
\end{tabular}

KD: Kawasaki disease; Flu: influenza; IVIG: immunoglobulin; IQ range: inter-quartile range; *T- test; \# Fisher's exact test. 
TABLE 2 - Laboratory values upon admission of the three groups.

\begin{tabular}{|c|c|c|c|c|}
\hline Laboratory values, mean (SD) & $\mathrm{KD}+$ Flu group & KD group & Flu group & $\mathrm{P}$ value \\
\hline $\mathrm{PMN}(\%)$ & $55.47(24.02)^{*}$ & $69.87(14.19)^{*}$ & $29.53(18.43)$ & $<0.01$ \\
\hline Lymphocytes (\%) & $34.87(22.57)^{*}$ & $26.04(12.62)^{\#}$ & $64.41(16.27)^{\text {*\# }}$ & $<0.01$ \\
\hline Platelet count $\left(10^{3} / \mu \mathrm{L}\right)$ & $402.33(51.75)^{*}$ & $319.7(158.04)$ & $251.47(70.06)^{*}$ & $<0.01$ \\
\hline $\operatorname{ALB}(g / d L)$ & $35.37(4.75)$ & $38.05(22.82)$ & $37.1(4.62)$ & 0.08 \\
\hline $\operatorname{ALT}(\mathrm{U} / \mathrm{L})$ & $70.93(83.50)$ & $95.73(89.45)$ & $80.93(65.78)$ & 0.38 \\
\hline $\operatorname{AST}(\mathrm{U} / \mathrm{L})$ & $51.8(29.39)$ & $55.27(51.92)$ & $56.13(28.6)$ & 0.47 \\
\hline
\end{tabular}

KD: Kawasaki disease; SD: standard deviation; Flu: influenza; WBC: white blood cell; PMN: polymorphonuclear leukocytes; CRP: C-reactive protein; ESR: erythrocyte sedimentation rate; ALB: albumin; ALT: alanine aminotransferase; AST: aspartate aminotransferase; CK-MB: creatine kinase isoenzyme; U/L: units per liter. The Kruskal-Wallis analysis was used for continuous variables; when the null hypothesis was rejected, we used the Bonferroni method for

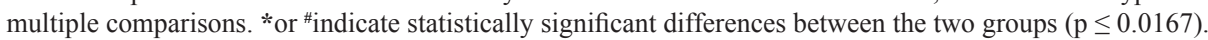

The influenza virus is a representative of the Orthomyxoviridae family and includes influenza viruses A, B and C. Mild and avirulent viruses infect only the throat and lungs because their hemagglutinin is only cleaved by proteases localized to the throat and lungs. Hemagglutinin can be cleaved by a wide variety of proteases in highly virulent strains, such as $\mathrm{H} 5 \mathrm{~N} 1$, allowing the virus to spread throughout the body. Influenza-infected cells produce large amounts of proinflammatory cytokines and chemokines, such as interferon and tumor necrosis factor ${ }^{(15)}$. This massive immune response can cause a life-threatening cytokine storm. Others have suggested that tumor necrosis factor can contribute to the formation of coronary lesions and coronary aneurysms ${ }^{(16)}$. These cytokines or inflammatory mediators can damage the vascular endothelium, which aggravates or causes $\mathrm{KD}$ coronary artery complications. In our study, the CRP and ESR values in the $\mathrm{KD}+$ Flu group were higher than those in the Flu group and $\mathrm{KD}$ group, which demonstrated that the manifestations of KD with concomitant influenza were not entirely due to the $\mathrm{KD}$ inflammatory response and that the influenza virus may play an important role in the total inflammatory response. Furthermore, the fever duration in the $\mathrm{KD}+$ Flu group was longer than other groups, indicating that more serious inflammation occurred in $\mathrm{KD}$ with concomitant influenza infection. Because of these factors, clinicians should be aware of the possibility of KD with concomitant influenza when a longer fever duration is observed in influenza or $\mathrm{KD}$ patients. Moreover, because patients in the $\mathrm{KD}$ + Flu group exhibited fewer principal clinical manifestations of $\mathrm{KD}$, the high CRP and ESR may help diagnose incomplete KD.

Influenza can exacerbate a number of diseases, including cardiovascular disease and diabetes, and can lead to viral pneumonia, secondary bacterial pneumonia or other viral/ bacterial infections. Influenza is a more common cause of mortality in cardiovascular disease patients than in patients with other chronic diseases ${ }^{(17)}$. Our results demonstrate that the CK-MB values in the Flu group were higher than in the KD group, and CK-MB abnormalities were observed more frequently in the KD + Flu group and Flu group than in the KD group. The coronary lesion rate was higher in the $\mathrm{KD}+$ Flu group than in the $\mathrm{KD}$ group (53.3\% vs. $40 \%)$. Based on the above evidence, we conclude that the influenza virus promotes cardiovascular damage in KD patients.

Previous studies have suggested that delayed therapy for $\mathrm{KD}$ or incomplete $\mathrm{KD}$ is associated with an increased risk of coronary artery aneurysms ${ }^{(18)(19)}$. In the present study, because KD patients with concomitant influenza infection too longer to diagnose, the fever duration before IVIG was longer for patients in the KD + Flu group than in the KD group. Patients often exhibited insufficient criteria for KD diagnosis, such as bilateral conjunctival injection, which was documented less frequently in KD patients with influenza, and this lack of criteria may have delayed the diagnosis. Additionally, this lack of criteria may explain why incomplete $\mathrm{KD}$ was observed more often in the $\mathrm{KD}+$ Flu group. In our study, although the coronary lesion rate was higher in the first group, we could not detect a significant difference between the KD + Flu group and the KD group. These potential differences may require additional study to confirm.

It is necessary to note that Reye's syndrome can occur in children who take salicylates while they are experiencing active infection with varicella or influenza; this condition has been reported in patients taking high-dose aspirin for a prolonged period of time after $\mathrm{KD}^{(20)}$. Kawasaki disease patients in this study were treated with aspirin, and the rate of Reye's syndrome was zero, but vigilance is still necessary. Other antiplatelet drugs, such as clopidogrel or dipyridamole, avoid such risk, but their effects should be studied more thoroughly.

In conclusion, influenza infection affects the clinical manifestations, laboratory test results, diagnosis, and treatment of $\mathrm{KD}$, but the potential contribution of influenza to KD etiology requires further study. 


\section{ACKNOWLEDGMENTS}

We would like to thank Guangzhou Medical College and Guangzhou Women and Children's Medical Center for providing support and guidance. The above opinions are those of the authors alone.

\section{CONFLICT OF INTEREST}

The authors declare that there is no conflict of interest.

\section{REFERENCES}

1. Bayers S, Shulman ST, Paller AS. Kawasaki disease: Part II. Complications and treatment. J Am Acad Dermatol 2013; 69:513. e1-513.e8.

2. Kawasaki T. Acute febrile mucocutaneous syndrome with lymphoid involvement with specific desquamation of the fingers and toes in children. Arerugi 1967; 16:178-222.

3. Park YW, Han JW, Park IS, Kim CH, Yun YS, Cha SH, et al. Epidemiologic picture of Kawasaki disease in Korea, 2000-2002. Pediatr Int 2005; 47:382-387.

4. Du ZD, Zhao D, Du J, Zhang YL, Lin Y, Liu C, et al. Epidemiologic study on Kawasaki disease in Beijing from 2000 through 2004. Pediatr Infect Dis J 2007; 26:449-451.

5. Jaggi P, Kajon AE, Mejias A, Ramilo O, Leber A. Human adenovirus infection in Kawasaki disease: a confounding bystander? Clin Infect Dis 2013; 56:58-64.

6. Leen C, Ling S. Mycoplasma infection and Kawasaki disease. Arch Dis Child 1996; 75:266-267.

7. Matsubara K, Fukaya T. The role of superantigens of group A Streptococcus and Staphylococcus aureus in Kawasaki disease. Curr Opin Infect Dis 2007; 20:298-303.

8. Nigro G, Midulla M. Retrovirus and Kawasaki disease. Lancet 1986; 2:1045.

9. Kikuta H, Matsumoto S, Osato T. Kawasaki Disease and EpsteinBarr Virus. Acta Paediatr Jpn 1991; 33:765-770.
10. Moreira A, Leite I, Baptista A, Osório Ferreira E. Kawasaki disease associated with parainfluenza type 3 virus infection. Acta Dermatovenerol Croat 2010; 18:120-123.

11. Newburger JW, Takahashi M, Gerber MA, Gewitz MH, Tani LY, Burns JC, et al. Diagnosis, treatment, and long-term management of Kawasaki disease: a statement for health professionals from the Committee on Rheumatic Fever, Endocarditis, and Kawasaki Disease, Council on Cardiovascular Disease in the Young, American Heart Association. Pediatrics 2004; 114:1708-1733.

12. Han RK, Sinclair B, Newman A, Silverman ED, Taylor GW, Walsh $\mathrm{P}$, et al. Recognition and management of Kawasaki disease. CMAJ 2000; 162:807-812.

13. Joshi AV, Jones KD, Buckley AM, Coren ME, Kampmann B. Kawasaki disease coincident with influenza A H1N1/09 infection. Pediatr Int 2011; 53:e1-e2.

14. Bayers S, Shulman ST, Paller AS. Kawasaki disease: Part I. Diagnosis, clinical features, and pathogenesis. J Am Acad Dermatol 2013; 69:501.e1-501.e11.

15. Schmitz N, Kurrer M, Bachmann MF, Kopf M. Interleukin-1 is responsible for acute lung immunopathology but increases survival of respiratory influenza virus infection. J Virol 2005; 79:6441-6448.

16. Hui-Yuen JS, Duong TT, Yeung RS. TNF- $\alpha$ is necessary for induction of coronary artery inflammation and aneurysm formation in an animal model of Kawasaki disease. J Immunol 2006; 176:6294-6301.

17. Glezen WP, Decker M, Perrotta DM. Survey of underlying conditions of persons hospitalized with acute respiratory disease during influenza epidemics in Houston, 1978-1981. Am Rev Respir Dis 1987; 136:550-555.

18. Tse SM, Silverman ED, McCrindle BW, Yeung RS. Early treatment with intravenous immunoglobulin in patients with Kawasaki disease. J Pediatr 2002; 140:450-455.

19. Berdej-Szczot E, Firek-Pędras M, Szydłowski L, KrzystolikŁadzińska J, Klimek K, Małecka-Tendera E. Analysis of risk factors and prospective evaluation of cardiovascular complications of Kawasaki disease in children: a single center study. Kardiol Pol 2013; 71:1279-1286.

20. Takahashi M, Mason W, Thomas D, Sinatra F. Reye syndrome following Kawasaki syndrome confirmed by liver histopathology in Kawasaki Disease. Paper presented at: Proceedings of the $5^{\text {th }}$ International Kawasaki Disease Symposium; May 22-25; Fukuoka, Japan; 1995. 